\title{
Metabolomic profiles of myocardial ischemia under treatment with salvianolic acid B
}

Yonghai Lu' ${ }^{1,3}$, Yue Zheng ${ }^{2,4}$, Xinru Liu' ${ }^{1}$ Xu Liang ${ }^{1}$, Saiming Ngai ${ }^{3}$, Tiejun $\mathrm{Li}^{2^{*}}$ and Weidong Zhang ${ }^{1^{*}}$

\begin{abstract}
Background: Radix Salvia miltiorrhiza (Danshen) has been used as a principal herb in treating cardiovascular diseases in Chinese medicine. Salvianolic acid B (SA-B), a water-soluble active component of Danshen, was found to have anti-myocardial ischemia (anti-MI) effect. This study aims to investigate mechanisms of SA-B on MI.

Methods: Five conventional Western medicines (isosorbide dinitrate, verapamil, propranolol, captopril and trimethazine) with different mechanisms for treating cardiovascular diseases were selected as positive references to compare with SA-B in changing of the metabolomic profiles in MI rats under treatment. Potential mechanisms of SA-B were further investigated in $\mathrm{H} 9 \mathrm{C} 2$ cell line.

Results: The metabolomic profiles between SA-B- and propranolol-treated MI rats were similar, since there was a big overlap between the two groups in the PLS-DA score plot. Finally, it was demonstrated that SA-B exhibited a protective effect on MI mainly by decreasing the concentration of cyclic adenosine monophosphate (CAMP) and $\mathrm{Ca}^{2+}$ and inhibiting protein kinase A (PKA).
\end{abstract}

Conclusion: SA-B and propanolol exhibited similar metabolomic profiles, indicating that the two drugs might have a similar mechanism.

\section{Background}

Myocardial ischemia (MI) is characterized by ischemia in the heart muscle. It is the most common cause of death, and a major reason for hospital admissions [1]. In MI, $\beta$-adrenoceptor is generally activated. Noradrenaline binds to $\beta$-adrenoceptor to activate GS protein. Then, adenylate cyclase (AC) is activated by GS protein, which makes adenosine triphosphate (ATP) to be cyclic adenosine monophosphate (cAMP). cAMP in turn activates the cAMP-dependent protein kinase A (PKA). This kinase phosphorylates several proteins related to excitation-contraction coupling, such as L-type $\mathrm{Ca}^{2+}$ channel and phospholamban. The phosphorylation of L-type $\mathrm{Ca}^{2}$ ${ }^{+}$channel causes the $\mathrm{Ca}^{2+}$ influx, leading to stronger muscle contraction. The phosphorylation of phospholamban accelerates $\mathrm{Ca}^{2+}$ uptake into the sarcoplasmic

\footnotetext{
* Correspondence: Itj204@163.com; wdzhangy@hotmail.com

'Department of Medicinal Chemistry of Nature Product, School of Pharmacy, Second Military Medical University, No. 325 Guohe Road, Shanghai 200433, China

${ }^{2}$ Department of Pharmacology, School of Pharmacy, Second Military Medical University, No. 325 Guohe Road, Shanghai 200433, China

Full list of author information is available at the end of the article
}

reticulum, increasing the rate and extent of muscle relaxation $[2,3]$.

$\beta$-adrenergic blockers such as propranolol [4] could inhibit the activation of $\beta$-adrenoceptor and decrease the concentration levels of cAMP, PKA and $\mathrm{Ca}^{2+}$, leading to slow heart rate, decreased myocardial contractility, reduced cardiac output, and decreased myocardial oxygen consumption. Besides, Western medicines with other mechanisms of action, such as isosorbide dinitrate (a vasodilator), verapamil (a calcium antagonist), captopril (an angiotensin converting enzyme inhibitor) and trimethazine (a fatty acid oxidation inhibitor), are also commonly used in the prevention and treatment of MI.

In recent years, radix salvia miltiorrhiza (Danshen) is also widely used in Chinese medicine for the treatment of cardiovascular diseases [5]. Danshen preparations in China medicine, including Compound Danshen Tablet, Compound Danshen Dripping Pill and Compound Danshen Granule [6-8] have been developed with interest from pharmaceutical industry.

Lam et al. $[9,10]$ found that the dilator action of Danshen on rat femoral artery was primarily produced by the inhibition of $\mathrm{Ca}^{2+}$ influx in the vascular smooth
C Biomed Central

(c) 2012 Lu et al; licensee BioMed Central Ltd. This is an Open Access article distributed under the terms of the Creative Commons Attribution License (http://creativecommons.org/licenses/by/2.0), which permits unrestricted use, distribution, and reproduction in any medium, provided the original work is properly cited. 
muscle cells. Kim et al. [11] found that Danshen could activate the endothelial nitric oxide synthase to induce vasodilation and reduce blood pressure. However, there is no final conclusion about mechanisms of Danshen. Thus, most scientists hope to increase understanding of mechanisms of Danshen by analyzing active components of Danshen. Presently, many active components have been isolated and identified in Danshen, such as tanshinone IIA, and salvianolic acid B (SA-B) [12-14].

SA-B, a water-soluble active component of Danshen, is effective for the protection of heart from ischemia [15]. Several possible cardio-protective effects were proposed, including augmenting vascular endothelial growth factor (VEGF) expression, promoting angiogenesis, recovering the normal expressions of sarco/endoplasmic reticulum ATPase $2 \mathrm{a}$ and phospholamban, and inhibiting the activation of platelet during myocardial ischemia and reperfusion [16-20].

Metabolomics is an emerging technique in the field of "omics" research and it is the systematic study of metabolites and its profile in a biological matrix, such as a cell, organ or organism [21,22]. It aims to pinpoint interesting metabolites that are related to disease or drug treatment. Verhoeckx et al. [23] successfully integrated the transcriptomic, proteomic and metabolomic techniques to characterize inflammatory modulators on the basis of their biological responses. Zilpaterol, a compound originally developed as a $\beta_{2}$-agonist but later specifically introduced as a growth-promoting agent, showed a pattern of mRNA and lipid expression almost identical to that showed by clenbuterol and salbutamol, $\beta_{2}$-agonists. In the same way, anti-MI drugs may be categorized into classes based on their mechanisms of action.

In this study, metabolomic approach was used to explore potential mechanisms of SA-B by comparing with five Western medicines (isosorbide dinitrate, verapamil, propranolol, captopril and trimethazine) conventionally used for the treatment of cardiovascular diseases.

\section{Methods}

\section{Materials}

HPLC grade acetonitrile was purchased from JT Baker (NJ, USA). Spectroscopic grade formic acid, leucine enkephalin, dimethyl sulfoxide (DMSO) and 3-(4, 5dimethylthiazol-2-yl)-2, 5-diphenyltetrazolium bromide were purchased from Sigma/Aldrich (MO, USA). Distilled water was purified "in-house" using a Milli-Q20 system Millipore (MA, USA). SA-B was purchased from the National Institute for the Control of Pharmaceutical and Biological Products (Beijing, China). Isosorbide dinitrate was purchased from Forward Co., Ltd (Shanghai, China). Verapamil was purchased from Shanghai
Pharmaceutical (Group) Co., Ltd (Shanghai, China). Propranolol was purchased from Shanghai Xinpashi Pharmaceutical Co., Ltd (Shanghai, China). Captopril was purchased from Shanghai Hengshan Pharmaceutical Factory (Shanghai, China). Trimethazine was purchased from Servier Pharmaceutical Factory (Tianjin, China). Fluo3/AM was purchased from Dojindo Laboratory (Tokyo, Japan). cAMP-Glo ${ }^{\mathrm{TM}}$ Assay and PepTag ${ }^{\circledR}$ NonRadio cAMP-Dependent Protein Kinase Assay were purchased from Promega (Madison, USA).

\section{Animals and drug administration}

Fifty male Sprague-Dawley rats (185-215 g) were purchased from the Slac Laboratory Animal Co., Ltd (Shanghai, China). The animals were housed in stainless steel metabolic cages under controlled conditions of humidity $(40-60 \%)$, temperature $\left(23-27^{\circ} \mathrm{C}\right)$, and a $12 \mathrm{~h}$ light-dark cycle. They were allowed free access to food and tap water. MI model was induced by left anterior descending coronary artery ligation [24]. The sham group rats were given no ligation. Electrocardiograms (ECG) were recorded by MPA 2000 Bio-signal Analysis System (Alcott Biotech Co. Ltd., Shanghai, China) to ascertain that the induction of MI was successful. Among 42 MI rats, 36 were arbitrarily selected and divided into six treatment groups $(\mathrm{n}=6)$ : SA-B $(20 \mathrm{mg} /$ $\mathrm{kg} / \mathrm{d})$, isosorbide dinitrate $(3 \mathrm{mg} / \mathrm{kg} / \mathrm{d})$, verapamil $(6$ $\mathrm{mg} / \mathrm{kg} / \mathrm{d})$, propranolol $(20 \mathrm{mg} / \mathrm{kg} / \mathrm{d})$, captopril $(4 \mathrm{mg} / \mathrm{kg} /$ d) and trimethazine $(6 \mathrm{mg} / \mathrm{kg} / \mathrm{d})$. In sham $(\mathrm{n}=6)$ and MI $(\mathrm{n}=6)$ groups, rats were received $0.2 \mathrm{~mL}$ of saline each time. Drugs were weighed and crushed into fine powder, which were orally administrated to rats with saline for 7 days after modeling. Rats were fasted overnight but with free access to water before their first administration. Blood samples were collected from ophthalmic venous plexus on the ninth day. The experiment was carried out in accordance with the guidelines of the Committee on the Care and Use of Laboratory Animals of the Institute of Laboratory Animal Resources of Shanghai, China. The Animal Care and Use Committee of Second Military Medical University also approved the study protocol.

\section{Cell culture}

The H9C2 cell line (Catalog No. GNR 5) was obtained from the Cell Bank of the Chinese Academy of Sciences and maintained in Dulbecco's Modified Eagle's Medium (DMEM; GIBCO, Paisley, UK) containing 10\% fetal bovine serum (FBS), penicillin-G $(100 \mathrm{U} / \mathrm{mL})$ and streptomycin $(100 \mathrm{U} / \mathrm{mL})$, and incubated in a humidified $\mathrm{CO}_{2}$ incubator (Sanyo, Japan) at $37^{\circ} \mathrm{C}$ with $5 \% \mathrm{CO}_{2}$. The cells were seeded at a density of $1 \times 10^{4} /$ well in a 96well plate, and cultured for $12 \mathrm{~h}$ for adherence. Cells were pre-treated with propranolol $(0.01 \mathrm{mg} / \mathrm{mL})$ or SA- 
$\mathrm{B}$ at concentrations of $0.001,0.01$ and $0.1 \mathrm{mg} / \mathrm{mL}$ for 2 $\mathrm{h}$, and the cells were incubated in a Tri-Gas $/ \mathrm{CO}_{2}$ incubator (Sanyo, Japan) at $37^{\circ} \mathrm{C}$ with $5 \% \mathrm{CO}_{2}$ and $95 \% \mathrm{~N}_{2}$ which was anoxic circumstance for $24 \mathrm{~h}$. The cells were incubated in a humidified $\mathrm{CO}_{2}$ incubator (Sanyo, Japan) at $37^{\circ} \mathrm{C}$ with $5 \% \mathrm{CO}_{2}$ as a control group. $\mathrm{Ca}^{2+}$, cAMP and PKA in the cells were measured. SA-B and propranolol were dissolved in DMSO. Appropriate amount of DMSO was also added to control media so that the same final concentration of DMSO (less than $0.1 \%$ ) can be kept in all groups.

\section{Metabolomics}

A blood sample $(1 \mathrm{~mL})$ from each rat was collected and kept in $2.5 \mathrm{~mL}$ heparin-coated tubes. These samples were centrifuged at $2789.1 \times g$ for $10 \mathrm{~min}$ at $4^{\circ} \mathrm{C}$, and the supernatants were collected. Acetonitrile $(300 \mu \mathrm{L})$ was added to the supernatant $(100 \mu \mathrm{L})$. The mixture was shaken vigorously for $30 \mathrm{~s}$ and centrifuged at 9562.5 $\times g$ for $10 \mathrm{~min}$ at $4^{\circ} \mathrm{C}$. After centrifugation, the supernatant was analyzed on an ACQUITYTM UPLC system coupled to a Micromass Q-Tof Micro ${ }^{\mathrm{TM}}$ (Waters MS Technologies, Manchester, UK) equipped with an electrospray ionization source. A $2.1 \mathrm{~mm}$ i.d. $\times 100 \mathrm{~mm}$ ACQUITY' $1.7 \mu \mathrm{m}$ column (Waters, Milford, MA, USA) was used. The column was maintained at $45^{\circ} \mathrm{C}$. Mobile phase A was formic acid (0.1\%) in water; mobile phase B was formic acid (0.1\%) in acetonitrile; injection volume was $5 \mu \mathrm{L}$; flow rate was $400 \mu \mathrm{L} / \mathrm{min}$. The gradient duration program was: $0-1.5 \mathrm{~min}, 5 \%$ of $\mathrm{B} ; 1.5-9$ min, $5-100 \%$ of $B ; 9-14$ min wash with $100 \%$ of $B$; and a 3 min recycle time. The parameters of mass detection were: desolvation gas at $400 \mathrm{~L} / \mathrm{h}$; cone gas at $20 \mathrm{~L} / \mathrm{h}$; desolvation temperature at $250^{\circ} \mathrm{C}$; source temperature at $100^{\circ} \mathrm{C}$; capillary voltage at $3000 \mathrm{~V}$; cone voltage at $30 \mathrm{~V}$; collision energy at $5 \mathrm{eV}$, while it was set at $20 \mathrm{eV}$ in MS/MS mode to identify potential biomarkers. The data acquisition rate was set as $0.4 \mathrm{~s}$, with $0.1 \mathrm{~s}$ inter-scan delay. Lock spray was used to calibrate accuracy of mass. Leucine enkephalin was used as the lock mass ( $\mathrm{m} / \mathrm{z} 556.2771$ in the positive mode, and 554.2615 in the negative mode). Data were collected in the continuum mode and averaged over 10 scans. The lock spray frequency was set at $10 \mathrm{~s}$. Full scan mass range of 50-1000 $\mathrm{m} / \mathrm{z}$ was acquired.

\section{Cell viability}

Cell viability was assessed by the MTT assay [25]. The cells were treated with SA-B at concentrations of $0.0001,0.001,0.01$ and $0.1 \mathrm{mg} / \mathrm{mL}$ for $24 \mathrm{~h}$. Then, the optical density (OD) values of samples were measured using a microplate reader (Thermo Lab systems, Shanghai, China) at $570 \mathrm{~nm}$ (630 $\mathrm{nm}$ as a reference).

\section{Measurement of calcium}

The $\mathrm{Ca}^{2+}$-sensitive was detected by dyeing with fluo-3/ AM [26]. The changes of $\mathrm{Ca}^{2+}$ in cells were examined by flow cytometry (BD Biosciences, USA).

\section{Measurement of cAMP}

The cAMP-Glo ${ }^{\mathrm{TM}}$ assay was used to monitor changes in cAMP concentrations [27]. The luminescence was measured by the plate-reading luminometer (Thermo Lab systems, Shanghai, China).

\section{Determination of PKA activity}

The activity of PKA was detected by non-radioactive PepTag PKA assay kit (Promega, Madison, USA) with dyelabeled Kemptide as substrate according to the manufacturer's protocol [28]. The cells were first homogenized in a cold PKA extraction buffer. After centrifugation, the supernatants were collected for the determination.

\section{Data processing}

The spectral data were exported by Micromass MarkerLynx ${ }^{\mathrm{TM}}$ applications manager version 4.1 software (Waters Corporation, Milford, MA, USA). The raw data of each sample was normalized to total area to correct for the MS response shift from the first injection to the last injection due to the long duration, overnight or longer, of an LC-MS analysis. The sum of the ion peak area within each sample was set at 10,000. After processing, partial least squares discriminant analysis (PLS-DA) was used for analysis of metabolite profiles, which was performed by the SIMCA-P software version 11 (Umetrics AB, Umeå, Sweden). In cell culture, each assay was performed in triplicate and repeated three times. The data were expressed as mean \pm standard deviation (SD). The significance was tested by one-way analyses of variance (ANOVA) of the SPSS 13.0 for Windows (SPSS Inc., Chicago, IL, USA), followed by the Duncan post hoc test. $P$ values less than 0.05 were considered significant.

\section{Results and discussion}

This study aims to investigate mechanisms of SA-B on MI. Firstly, metabolomics in combination with multivariate statistical analysis was used to display potential mechanisms of SA-B by comparing with five Western medicines (isosorbide dinitrate, verapamil, propranolol, captopril and trimethazine) conventionally used for the treatment of cardiovascular diseases. Secondly, the potential mechanisms of SA-B suggested by the metabolomic study were further investigated in $\mathrm{H} 9 \mathrm{C} 2$ cell line.

\section{Metabolomics}

In our previous study [29], a total of 2700 ions were detected in metabolic profile of rats; 160 out of 2700 
ions were significantly changed in MI rats as compared with sham rats; 22 out of 160 ions were identified as biomarkers of MI, including 15(S)-HETE, 2',3'-Cyclic AMP, dihydrosphingosine, 5-phosphoribosylamine, phytosphingosine, L-isoleucyl-L-proline, 2',3'-Cyclic GMP, 1-phenylethylamine, thromboxane B2, xanthine, hypoxanthine, inosine, L-homoserine, carnosine, allantoin, Lvaline, L-phenylalanine, dihydrobiopterin, 2-oxoisocaproic acid, L-isoleucine, L-tryptophan and glyceraldehydes. In addition, SA-B was also found to be effective for treating MI [8]. The present study revealed the antiMI effect of SA-B in more detail.

\section{Anti-myocardial ischemia effect of SA-B}

PLS-DA model [29] was built based on the significantly changed ions (Top 160) observed in MI to evaluate the protective effects of SA-B on MI. The PLS-DA score plot (Figure 1A) showed that sham, MI and SA-B-treated rats were classified, and the SA-B-treated rats were much closer to the sham rats than the MI rats, suggesting that SA-B had a positive impact on repairing the abnormal metabolic profiles induced by MI. To validate the result statistically, the PLS-DA model was validated by permutation test $(\mathrm{n}=100)$ (Figure $1 \mathrm{~B})$. Generally, $\mathrm{Q}^{2}$-intercept in permutation test should be less than

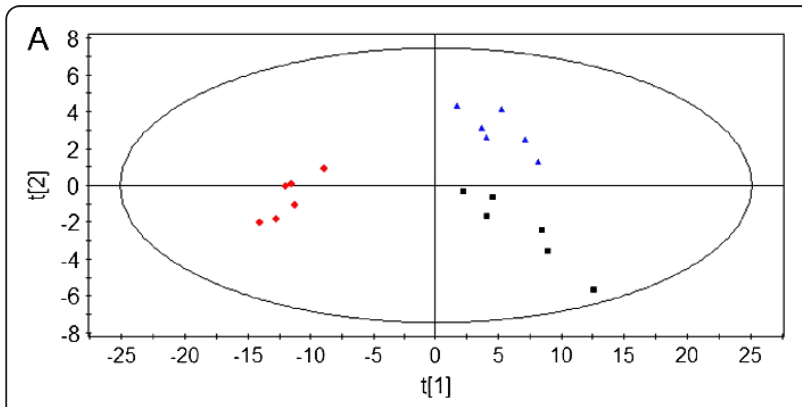

B

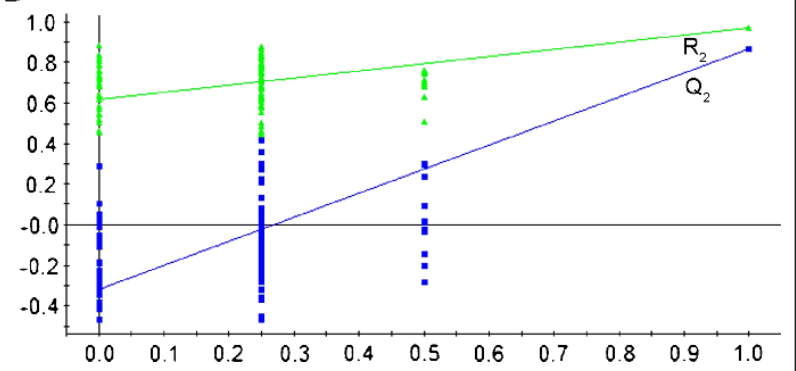

Figure 1 (a) PLS-DA score plot of the metabolic profiles obtained from 6 sham rats $(\varpi), 6 \mathrm{MI}$ rats with no treatment (red diamond symbol), and $6 \mathrm{MI}$ rats with SA-B treatment (blue triangle symbol). $\left(R^{2} X=0.798, R^{2} Y=0.975, Q^{2}=0.858, A=4, N=\right.$ $18, K=160$ ); (b) Validation plot obtained from permutation test $(n=$ 100). $R^{2}$ is the explained variance, and $Q^{2}$ is the predictive ability of the model.
0.05 . The intercept value of $\mathrm{Q}^{2}$ for this model was -0.315 , which meant no overfitting.

The mean levels of 22 identified MI biomarkers in SA-B-treated rats were calculated to further evaluate the regulations of SA-B. As shown in Table 1, levels of 18 biomarkers in SA-B-treated rats were statistically reversed by compared with those in MI rats $(P<0.05$, SA-B-treated groups versus MI group) [8]. The result indicated that SA-B could regulate the abnormal metabolites induced by MI.

\section{Potential mechanisms of SA-B}

In order to investigate the mechanisms of SA-B, we selected five Western medicines (isosorbide dinitrate, verapamil, propranolol, captopril and trimethazine) with different mechanisms for treating MI as positive references. PLS-DA model [29] was established based on the global metabolomic profile of MI rats to compare the influences of SA-B and five Western medicines. The PLS-DA score plot (Figure 2) showed five Western medicine-treated groups were separated and the SA-B-treated group overlapped with the propranolol-treated group (each symbol in the plot represents a sample), indicating that the metabolomic profile of SA-B-treated rats was similar with that of propranolol-treated rats. Model validation with permutation test $(\mathrm{n}=100)$ generated intercepts of $Q^{2}=-0.301$, which suggested there was no overfitting. The result indicated that SA-B and propanolol might have a similar mechanism in treating MI, since the drugs with the same mechanism showed similar effects on the metabolomic profile [23].

By monitoring the changes of the 22 biomarkers after administration, the regulations of SA-B and propranolol were further compared. As aforementioned, 18 biomarkers were regulated by SA-B $(P<0.05$, SA-B-treated group versus MI group) (Table 1). Herein, we found that levels of 21 biomarkers were reversed in propranolol-treated rats by compared with those in MI rats $(P<$ 0.05 , propranolol-treated group versus MI group) (Table 1) [29]. The results further suggested that SA-B and propranolol had similar regulations on biomarkers of MI rats, indicating they might have a similar mechanism.

\section{Validation of mechanisms of SA-B in $\mathrm{H} 2 \mathrm{C} 2$ cell line}

As aforementioned, propranolol [4] could inhibit the activation of $\beta$-adrenoceptor and decrease the concentration levels of cAMP, PKA and $\mathrm{Ca}^{2+}$, leading to slow heart rate, decreased myocardial contractility, reduced cardiac output, and decreased myocardial oxygen consumption. In order to prove the propranolol-like effect of SA-B, we tested whether SA-B could block $\beta$-adrenoceptor directly. Unfortunately, specific binding of SA-B to $\beta$-adrenoceptor was not found in our early study (not 
Table 1 Beneficial effects of SA-B and propranolol on MI biomarkers

\begin{tabular}{|c|c|c|c|c|c|c|}
\hline \multirow[t]{2}{*}{ Biomarkers } & \multirow{2}{*}{$\begin{array}{l}\text { Sham group } \\
\text { Mean } \pm S D^{a}\end{array}$} & \multirow{2}{*}{$\begin{array}{c}\text { MI group } \\
\text { Mean } \pm \text { SD }\end{array}$} & \multirow{2}{*}{$\begin{array}{l}\text { SA-B group } \\
\text { Mean } \pm \text { SD }\end{array}$} & \multirow{2}{*}{$\begin{array}{c}\text { Propranolol group } \\
\text { Mean } \pm \text { SD }\end{array}$} & \multicolumn{2}{|c|}{$P$ value } \\
\hline & & & & & SA-B vs. MI & Propranolol vs. MI \\
\hline Allantoin & $23.87 \pm 4.14$ & $0.00 \pm 0.00$ & $20.32 \pm 5.36$ & $23.16 \pm 8.17$ & 0.000 & 0.000 \\
\hline 1-Phenylethylamine & $4.13 \pm 1.88$ & $23.66 \pm 1.33$ & $12.61 \pm 0.96$ & $9.42 \pm 1.36$ & 0.001 & 0.000 \\
\hline Hypoxanthine & $0.00 \pm 0.00$ & $14.72 \pm 14.01$ & $6.68 \pm 2.86$ & $0.00 \pm 0.00$ & 0.001 & 0.000 \\
\hline L-Valine & $37.98 \pm 3.80$ & $13.17 \pm 2.14$ & $22.75 \pm 4.25$ & $29.41 \pm 6.31$ & 0.002 & 0.001 \\
\hline L-Isoleucine & $122.84 \pm 21.91$ & $39.18 \pm 2.37$ & $92.59 \pm 12.26$ & $139.28 \pm 26.48$ & 0.001 & 0.000 \\
\hline Carnosine & $16.18 \pm 2.70$ & $0.00 \pm 0.00$ & $22.13 \pm 04.27$ & $19.35 \pm 2.34$ & 0.000 & 0.000 \\
\hline L-isoleucyl-L-Proline & $28.69 \pm 3.90$ & $79.23 \pm 21.02$ & $52.15 \pm 14.06$ & $41.32 \pm 20.49$ & 0.027 & 0.011 \\
\hline Phytosphingosine & $16.74 \pm 1.89$ & $82.93 \pm 10.12$ & $32.17 \pm 6.29$ & $41.38 \pm 5.44$ & 0.000 & 0.000 \\
\hline Dihydrosphingosine & $26.02 \pm 0.99$ & $146.98 \pm 15.82$ & $52.18 \pm 11.40$ & $31.37 \pm 7.14$ & 0.001 & 0.000 \\
\hline 2',3'-Cyclic GMP & $6.30 \pm 0.81$ & $37.05 \pm 5.97$ & $15.16 \pm 3.49$ & $11.32 \pm 2.40$ & 0.001 & 0.000 \\
\hline 2,,3'-Cyclic AMP & $30.33 \pm 1.78$ & $190.63 \pm 16.91$ & $153.21 \pm 19.48$ & $51.43 \pm 8.46$ & 0.008 & 0.000 \\
\hline Dihydrobiopterin & $42.64 \pm 4.78$ & $8.23 \pm 3.82$ & $19.18 \pm 8.31$ & $39.27 \pm 8.19$ & 0.015 & 0.000 \\
\hline Xanthine & $0.00 \pm 0.00$ & $25.94 \pm 6.39$ & $24.13 \pm 7.51$ & $0.00 \pm 0.00$ & 0.805 & 0.000 \\
\hline Inosine & $0.00 \pm 0.00$ & $12.84 \pm 3.49$ & $12.03 \pm 0.89$ & $0.00 \pm 0.00$ & 0.913 & 0.000 \\
\hline Glyceraldehyde & $189.71 \pm 13.83$ & $31.23 \pm 2.72$ & $99.30 \pm 9.47$ & $159.29 \pm 12.69$ & 0.000 & 0.000 \\
\hline L-Phenylalanine & $14.65 \pm 4.69$ & $0.00 \pm 0.00$ & $5.34 \pm 2.06$ & $9.28 \pm 2.44$ & 0.000 & 0.000 \\
\hline L-Tryptophan & $64.74 \pm 12.98$ & $20.06 \pm 1.15$ & $77.27 \pm 2.40$ & $59.45 \pm 5.46$ & 0.000 & 0.000 \\
\hline 2-oxoisocaproic acid & $27.85 \pm 3.98$ & $6.13 \pm 1.23$ & $17.33 \pm 3.54$ & $29.75 \pm 4.94$ & 0.000 & 0.000 \\
\hline 5-Phosphoribosylamine & $87.81 \pm 4.96$ & $211.14 \pm 15.24$ & $203.47 \pm 10.26$ & $129.32 \pm 24.94$ & 0.871 & 0.002 \\
\hline 15(S)-HETE & $0.00 \pm 0.00$ & $243.89 \pm 99.98$ & $71.67 \pm 4.40$ & $0.00 \pm 0.00$ & 0.001 & 0.000 \\
\hline Thromboxane B2 & $2.87 \pm 1.95$ & $18.27 \pm 2.92$ & $11.03 \pm 0.64$ & $9.42 \pm 3.38$ & 0.002 & 0.001 \\
\hline
\end{tabular}

${ }^{a}$ Mean peak areas of Ml biomarkers in different groups were calculated to reveal the beneficial effects of SA-B and propranolol; SD denotes standard deviation.

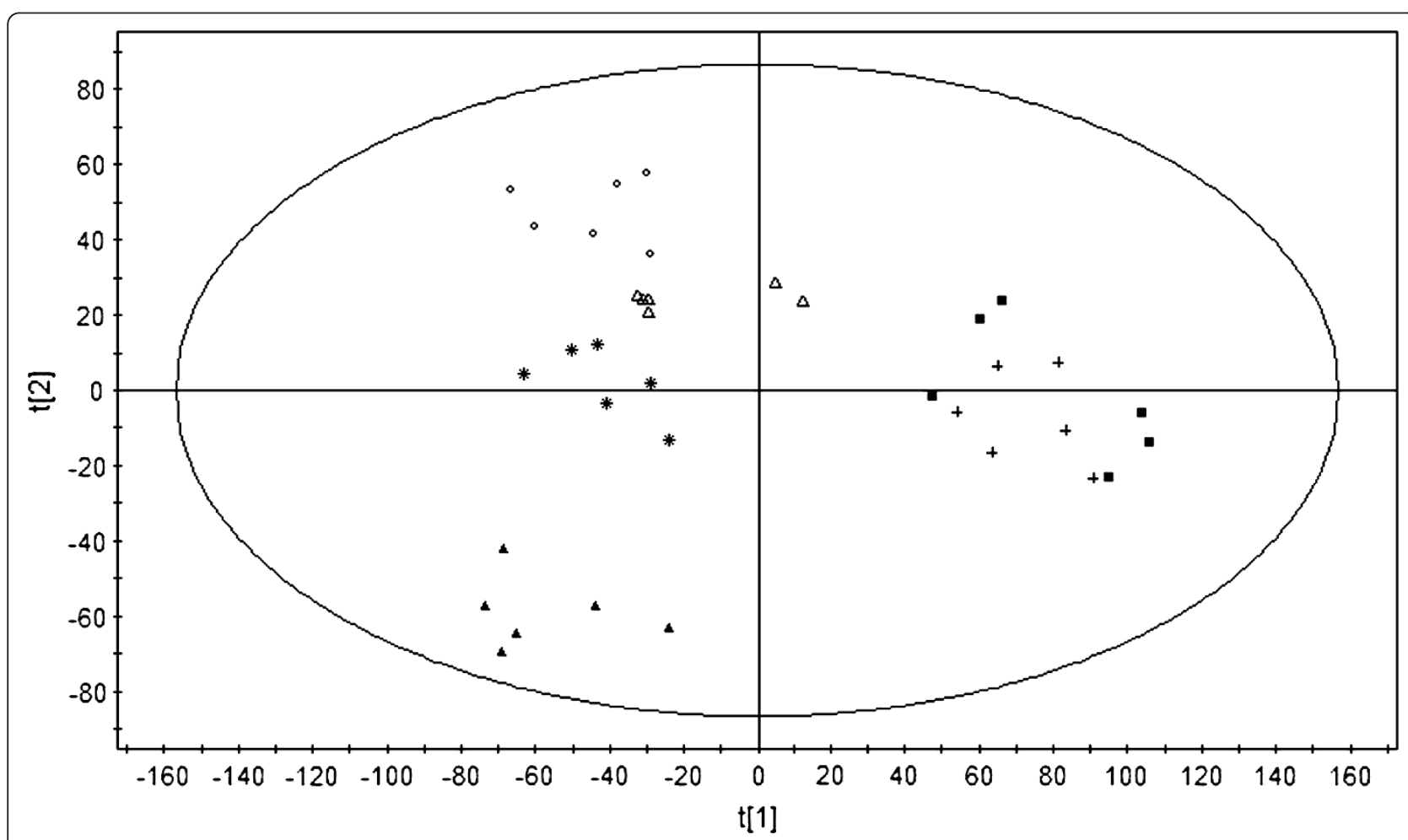

Figure 2 PLS-DA score plot of the metabolic profiles obtained from all drug-treated rats: $(+)$ SA-B, ( $(\square)$ propranolol $(\Delta)$ isosorbide dinitrate, $(0)$ trimethazine, $(*)$ verapamil, and $(\mathbf{\Delta})$ captopril. $\left(R^{2} Y=0.61, R^{2} X=0.833, Q^{2}=0.512, A=5, N=36, K=2700\right)$. 
published). In this study, we focused on the influences of SA-B and propranolol on the downstream regulation in $\beta$-adrenoceptor signaling pathway. The effects of SA$\mathrm{B}$ on the levels of on cAMP, PKA and $\mathrm{Ca}^{2+}$ in cells were investigated by $\mathrm{H} 9 \mathrm{C} 2$ cell line, which is a commercially available myogenic cell line derived from embryonic rat heart ventricle [30] and is widely accepted as a substitute for rat cardiomyocyte in in vitro study [31].

The viability of $\mathrm{H} 9 \mathrm{C} 2$ cells was not affected significantly by pretreatment with SA-B up to $0.1 \mathrm{mg} / \mathrm{mL}$ for $24 \mathrm{~h}$ (Figure 3 ), indicating that SA-B did not cause damage to $\mathrm{H} 9 \mathrm{C} 2$ cells. The results of $\mathrm{Ca}^{2+}$, cAMP and PKA assays showed that SA-B significantly decreased $\mathrm{Ca}^{2+}$ concentration in anoxic $\mathrm{H} 9 \mathrm{C} 2$ cells (Figure 4). We suggested that SA-B might have a strong effect on the regulation of $\mathrm{Ca}^{2+}$ concentration even at a very low dosage $(0.001 \mathrm{mg} / \mathrm{mL})$. We also found that both SA-B and proprannolol significantly inhibited the increased levels of cAMP induced by anoxia (Figure 5). SA-B decreased cAMP concentration in a concentrationdependent manner. As shown in Figure 6, PKA activity was increased in anoxic cells, but significantly decreased by SA-B and proprannolol treatment. In addition, there was also no significant difference among these three SAB-treated groups. These results showed that SA-B could decrease the concentration of $\mathrm{Ca}^{2+}$ and inhibit the activation of PKA induced by anoxia at a very low dosage $(0.001 \mathrm{mg} / \mathrm{mL})$, but required a high dosage $(0.1 \mathrm{mg} / \mathrm{mL})$ to decrease the concentration of cAMP. The cell experiments demonstrated that SA-B had a similar role in regulation of concentrations of $\mathrm{Ca}^{2+}$, cAMP and activity of PKA as proprannolol.

Our previous studies suggested that the metabolomic profile was changed in MI rats [29], and SA-B could reverse the levels of most MI biomarkers [8]. The present study indicated that SA-B- and propanolol-treated rats had similar metabolomic profiles, which suggested that the two drugs might have a similar mechanism in treating MI, since the drugs with the same mechanism showed similar effects on the metabolomic profile. The hypothesis that SA-B and propranolol had a similar mechanism in treating MI was supported by these experiments. Although no evidence showed that SA-B

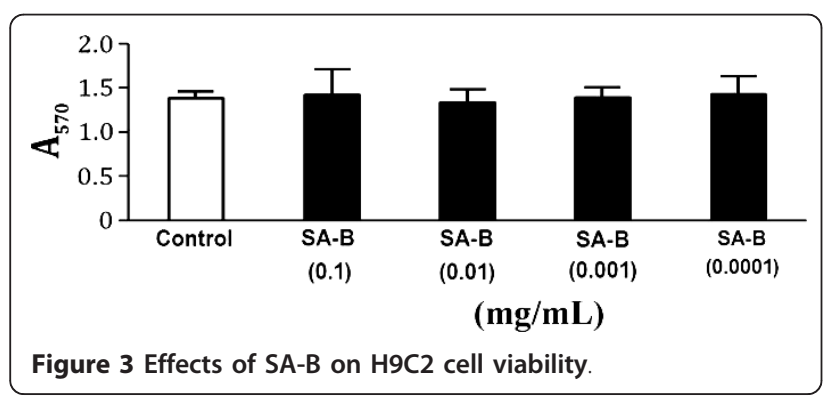

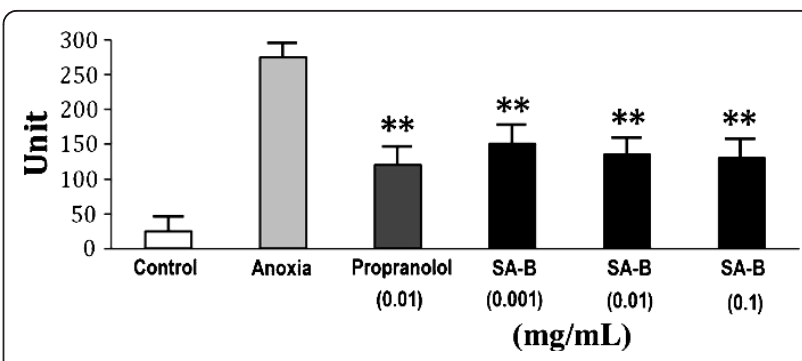

Figure 4 Effects of SA-B on the cellular calcium concentration in $\mathrm{H} 9 \mathrm{C} 2$ cells. ${ }^{* *} P<0.01$ compared with anoxia.

could block $\beta$-adrenoceptor directly, the cell experiments demonstrated that SA-B showed a similar role in regulation of concentrations of $\mathrm{Ca}^{2+}$, cAMP and activity of PKA as proprannolol. While the drug action of the metabolites of SA-B is still under investigation, our results suggest that metabolomics is a good analytical method for understanding mechanisms of SA-B.

\section{Conclusions}

SA-B is a water-soluble active component of Danshen, and is potentially effective for the protection of heart from ischemia-reperfusion. Metabolomic results showed that SA-B and propranolol had similar effects on metabolic profiles of MI rats. The cell experiments

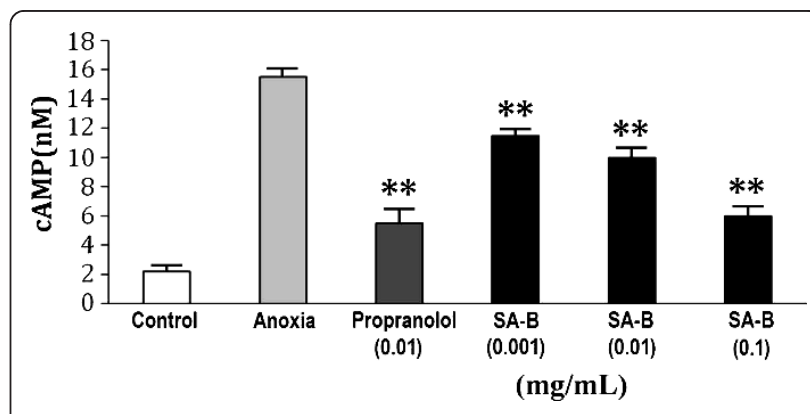

Figure 5 Effects of SA-B on the levels of cAMP in H9C2 cells. ${ }^{*} P<0.01$ compared with anoxia.

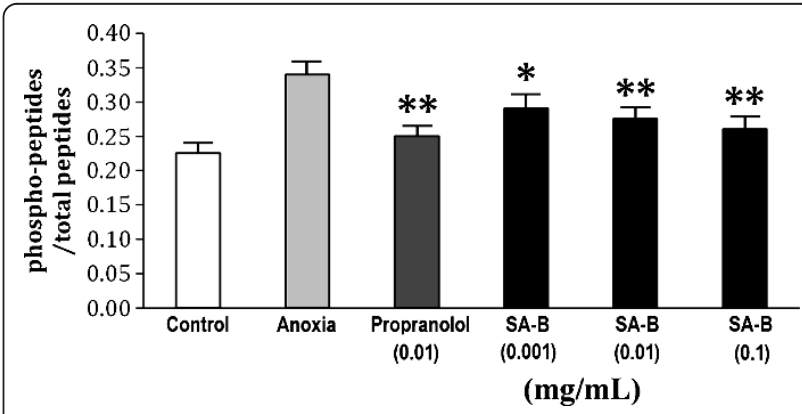

Figure 6 Effects of SA-B on the PKA activity in H9C2 cell. ${ }^{* *} P<$ 0.01 compared with anoxia. 
demonstrated that SA-B had protective effects on MI mainly by decreasing $\mathrm{Ca}^{2+}$ and cAMP and inhibiting the activation of PKA. This study indicates that SA-B and propanolol might have a similar mechanism in treating MI.

\section{Abbreviations}

Ml: myocardial ischemia; AC: Adenylate cyclase; ATP: adenosine triphosphate; CAMP: cyclic adenosine monophosphate; PKA: protein kinase A; SA-B: salvianolic acid B; VEGF: vascular endothelial growth factor; DMSO: dimethyl sulfoxide; ECG: electrocardiograms; DMEM: Dulbecco's Modified Eagle's Medium; FBS: fetal bovine serum; OD: optical density value; PLS-DA: partial least squares discriminant analysis; SD: standard deviation; ANOVA: one-way analyses of variance.

\section{Acknowledgements}

The work was supported by program NCET Foundation, NSFC (30725045), the Special Program for New Drug Innovation of the Ministry of Science and Technology, China (2009ZX09311-001, 2008ZX09308-005), Shanghai Leading Academic Discipline Project (B906) and in part by the Scientific Foundation of Shanghai China (09DZ1975700, 09DZ1971500).

\section{Author details}

'Department of Medicinal Chemistry of Nature Product, School of Pharmacy, Second Military Medical University, No. 325 Guohe Road, Shanghai 200433, China. ${ }^{2}$ Department of Pharmacology, School of Pharmacy, Second Military Medical University, No. 325 Guohe Road, Shanghai 200433, China. ${ }^{3}$ School of Life Sciences, Faculty of Science, The Chinese University of Hong Kong, Hong Kong, China. ${ }^{4}$ Institute for Drug and Instrument Control of Health Department, General Logistics Department of the Chinese People's Liberation Army, Beijing 100071, China.

\section{Authors' contributions}

YHL, YZ, TJL and WDZ designed the study. YHL conducted the metabolomic study and wrote the manuscript. $Y Z$ assisted in the cell experiments and in writing the manuscript. XRL and $X L$ assisted in writing the manuscript. SMN, TJL and WDZ amended the manuscript. All authors read and approved the final manuscript.

\section{Competing interests}

The authors declare that they have no competing interests.

Received: 12 November 2011 Accepted: 13 March 2012 Published: 13 March 2012

\section{References}

1. World Health Organization: Department of Health Statistics and Informatics in the Information, Evidence and Research Cluster. The global burden of disease 2004 update. Geneva: WHO; 2004.

2. Gao Y, Gao G, Long C, Han S, Zu P, Fang L, Li J: Enhanced phosphorylation of cyclic AMP response element binding protein in the brain of mice following repetitive hypoxic exposureal. Biochem Biophys Res Commun 2006, 340:661-667.

3. Leineweber K, Böhm M, Heusch G: Cyclic adenosine monophosphate in acute myocardial infarction with heart failure: slayer or savior? Circulation 2006, 114:365-367.

4. Chakraborti S, Das S, Kar P, Ghosh B, Samanta K, Kolley S, Ghosh S, Roy S, Chakraborti T: Calcium signaling phenomena in heart diseases: a perspective. Mol Cell Biochem 2007, 29:1-40.

5. Lin $T H$, Hsieh CL: Pharmacological effects of Salvia miltiorrhiza (Danshen) on cerebral infarction. Chin Med 2010, 5:2.

6. Davidson P, Hancock K, Leung D, Ang E, Chang E, Thompson DR, Daly J: Traditional Chinese medicine and heart disease: what does Western medicine and nursing science know about it? Eur J Cardiovasc Nurs 2003, 2:171-181.

7. Editorial Committee of Pharmacopoeia of Ministry of Health P.R. China: The Pharmacopoeia of People's Republic of China. Chinese Medical Science and Technology Press, Beijing; 2010, year 2010.
8. Lu YH, Liu XR, Liang X, Xiang L, Zhang WD: Metabolomic strategy to study therapeutic and synergistic effects of tanshinone IIA, salvianolic acid B and ginsenoside Rb1 in myocardial ischemia rats. J Ethnopharmacol 2011, 134:45-49.

9. Lam FF, Yeung JH, Cheung JH: Mechanisms of the dilator action of Danshen (Salvia miltiorrhiza) on rat isolated femoral artery. J Cardiovasc Pharmacol 2005, 46:361-368.

10. Lam FF, Yeung JH, Cheung JH, Or PM: Pharmacological evidence for calcium channel inhibition by danshen (Salvia miltiorrhiza) on rat isolated femoral artery. J Cardiovasc Pharmacol 2006, 47:139-145.

11. Kim DD, Sánchez FA, Durán RG, Kanetaka T, Durán WN: Endothelial nitric oxide synthase is a molecular vascular target for the Chinese herb Danshen in hypertension. Am J Physiol Heart Circ Physiol 2007, 292: H2131-H2137.

12. Chen $Y$, Duan $G$, Xie M, Chen B, Li Y: Infrared-assisted extraction coupled with high-performance liquid chromatography for simultaneous determination of eight active compounds in Radix Salviae miltiorrhizae. J Sep Sci 2010, 33:2888-2897.

13. Sun Y, Zhu H, Wang J, Liu Z, Bi J: Isolation and purification of salvianolic acid A and salvianolic acid B from Salvia miltiorrhiza by high-speed counter-current chromatography and comparison of their antioxidant activity. J Chromatogr B Analyt Technol Biomed Life Sci 2009, 877:733-737.

14. Zhao J, Lou J, Mou Y, Li P, WU J, Zhou L: Diterpenoid tanshinones and phenolic acids from cultured hairy roots of Salvia miltiorrhiza Bunge and their antimicrobial activities. Molecules 2011, 16:2259-2267.

15. Feng $L X$, Jing $C J$, Tang KL, Tao L, Cao ZW, Wu WY, Guan SH, Jiang BH, Yang M, Liu X, Guo DA: Clarifying the signal network of salvianolic acid B using proteomic assay and bioinformatic analysis. Proteomics 2011, 11:1473-1485.

16. Fu J, Huang H, Liu J, Pi R, Chen J, Liu P: Tanshinone IIA protects cardiac myocytes against oxidative stress-triggered damage and apoptosis. Eur J Pharmacol 2007, 568:213-221.

17. He HB, Yang XZ, Shi MQ, Zeng XW, Wu LM, Li LD: Comparison of cardioprotective effects of salvianolic acid $B$ and benazepril on large myocardial infarction in rats. Pharmacol Rep 2008, 60:369-381.

18. Wang Z, Li M, Wu WK, Tan HM, Geng DF: Ginsenoside Rb1 preconditioning protects against myocardial infarction after regional ischemia and reperfusion by activation of phosphatidylinositol-3-kinase signal transduction. Cardiovasc Drugs Ther 2008, 22:443-452.

19. Xu W, Yang J, Wu LM: Cardioprotective effects of tanshinone IIA on myocardial ischemia injury in rats. Pharmazie 2009, 64:332-336.

20. Yang FG, Zhang AY, Chen ZY, Lian ZX, Liu GX, Dong GX: Effects of salvianolic acid $B$ on cardiovascular endothelial cells and platelet activation in a rabbit model of ischemia-reperfusion. Zhong $X_{i} Y_{i}$ Jie He Xue Bao 2008, 6:1250-1254.

21. Nicholson JK, Lindon JC, Holmes E: 'Metabonomics': understanding the metabolic responses of living systems to pathophysiological stimuli via multivariate statistical analysis of biological NMR spectroscopic data. Xenobiotica 1999, 29:1181-1118.

22. Slupsky CM, Steed H, Wells TH, Dabbs K, Schepansky A, Capstick V, Faught W, Sawyer MB: Urine metabolite analysis offers potential early diagnosis of ovarian and breast cancers. Clin Cancer Res 2010, 16:5835-5841.

23. Verhoeckx KC, Bijlsma S, Jespersen S, Ramaker R, Verheij ER, Witkamp RF, van der Greef J, Rodenburg RJ: Characterization of anti-inflammatory compounds using transcriptomics, proteomics, and metabolomics in combination with multivariate data analysis. Int Immunopharmacol 2004 4:1499-1514.

24. Ytrehus K: Models of myocardial ischemia. Drug Discov Today Dis Models 2006, 3:263-271.

25. Mosmann T: Rapid colorimetric assay for cellular growth and survival: application to proliferation and cytotoxicity assays. J Immunol Methods 1983, 65:55-63.

26. Bradley KN, Craig JW, Muir TC, McCarron JG: The sarcoplasmic reticulum and sarcolemma together form a passive $\mathrm{Ca}^{2+}$ trap in colonic smooth muscle. Cell Calcium 2004, 36:29-41.

27. Kumar M, Hsiao K, Vidugiriene J, Goueli SA: A bioluminescent-based, HTS compatible assay to monitor G-protein-coupled receptor modulation of cellular cyclic AMP. Assay Drug Dev Technol 2007, 5:237-245.

28. Saudohar M, Bencina M, van de Vondervoort PJ, Panneman H, Legisa $M$, Visser J, Ruijter GJ: Cyclic AMP-dependent protein kinase is involved in morphogenesis of Aspergillus niger. Microbiology 2002, 148:2635-2645. 
29. Lv YH, Liu XR, Yan SK, Liang X, Yang Y, Dai WX, Zhang WD: Metabolomic study of myocardial ischemia and intervention effects of Compound Danshen Tablets in rats using ultra-performance liquid chromatography/ quadrupole time-of-flight mass spectrometry. J Pharm Biomed Anal 2010, 52:129-135.

30. Kimes BW, Brandt BL: Properties of a clonal muscle cell line from rat heart. Exp Cell Res 1976, 98:367-381.

31. Zhou L, Ma W, Yang Z, Zhang F, Lu L, Ding Z, Ding B, Ha T, Gao X, Li C: VEGF165 and angiopoietin-1 decreased myocardium infarct size through phosphatidylinositol-3 kinase and Bcl-2 pathways. Gene Ther 2005, 12:196-202.

doi:10.1186/1749-8546-7-6

Cite this article as: Lu et al: Metabolomic profiles of myocardial

ischemia under treatment with salvianolic acid B. Chinese Medicine 2012

7:6.

Submit your next manuscript to BioMed Central and take full advantage of:

- Convenient online submission

- Thorough peer review

- No space constraints or color figure charges

- Immediate publication on acceptance

- Inclusion in PubMed, CAS, Scopus and Google Scholar

- Research which is freely available for redistribution

Submit your manuscript at www.biomedcentral.com/submit 